Bio - grafía. Escritos sobre la Biología y su Enseñanza. ISSN 2027-1034

Edición Extraordinaria. p.p. $36-43$

Memorias del IX Encuentro Nacional de Experiencias en Enseñanza de la Biología y la Educación Ambiental. IV Congreso Nacional de Investigación en Enseñanza de la Biología.

\title{
LA EXPERIENCIA EN LA PRÁCTICA INVESTIGATIVA: UNA DISCUSIÓN EN EL MARCO DE UNA PROPUESTA DE ESTRATEGIA PEDAGÓGICA DEL MUSEO DE BIOLOGÍA.
}

\begin{abstract}
THE EXPERIENCE IN THE INVESTIGATIVE PRACTICE: A DISCUSSION IN THE FRAMEWORK OF APROPOSED PEDAGOGICAL STRATEGY AROUND THE BIOLOGICAL COLLECTIONS AND THE CONFIGURATION OF THE BIOLOGY MUSEUM
\end{abstract}

\section{Ludvison Miguel Herrera Urrego ${ }^{1}$}

\section{RESUMEN}

El presente texto dialoga en relación a las discusiones que surgen a propósito de la participación en la propuesta de investigación Estrategia para la enseñanza de la vida y lo vivo a partir de las colecciones biológicas: Hacia la configuración del Museo Pedagógico. Situación desde donde emerge el acercamiento a figuras como estrategia pedagógica, colecciones biológicas y la vida y lo vivo. Éstas, si bien gozan de amplia movilización en el ámbito del Departamento de Biología DBI, versan en muchos casos en sinonimias entre otras figuras cercanas, dicotomías y múltiples alegorías en sus significaciones

Este motivante lleva a preguntarse por la contemplación de estas figuras como parte del ejercicio investigativo, en este sentido los siguientes párrafos discurren alrededor de su funcionalidad y la reflexión emergente. Vale aclarar que el objetivo de este texto dista de asegurar una posición adecuada o más válida en relación a las figuras mencionadas, por el contrario, su objetivo se halla en presentar las

\section{ABSTRACT.}

The present text talks about the discussions that arise regarding the participation in the research proposal Strategy for teaching life and living it from the biological collections: Towards the configuration of the Pedagogical Museum. Situation from where the approach to figures emerges as pedagogical strategy, biological collections and life and the living. Although they are widely mobilized within the DBI Department of Biology, they are in many cases synonymous with other nearby figures, dichotomies and multiple allegories in their meanings

This motivator leads to questioning the contemplation of these figures as part of the investigative exercise, in this sense the following paragraphs run around its functionality

\footnotetext{
${ }^{1}$ Licenciado en Biología, Monitor del proyecto de investigación Estrategia pedagógica para la enseñanza de la vida y lo vivo a partir de las colecciones biológicas: Hacia la configuración del Museo Pedagógico de Biología. Participante del grupo de investigación CASCADA.
} 
Bio - grafía. Escritos sobre la Biología y su Enseñanza. ISSN 2027-1034

Edición Extraordinaria. p.p. $36-43$

Memorias del IX Encuentro Nacional de Experiencias en Enseñanza de la Biología y la

Educación Ambiental. IV Congreso Nacional de Investigación en Enseñanza de la

Biología.

and emerging reflection. It is worth clarifying that the objective of this text is far from ensuring a proper or more valid position in relation to the mentioned figures, on the contrary, its objective is to present the views that permeate the development of research.

\section{PALABRAS CLAVE.}

Colección biológica, Estrategia pedagógica, Estrategia didáctica, Práctica de investigación.

\section{KEY WORDS}

Biological collection, Pedagogical strategy, Didactic strategy, Research practice

\section{INTRODUCCIÓN.}

El programa de Licenciatura en Biología de la Universidad Pedagógica Nacional se caracteriza por su intencionalidad frente a la formación de maestros que reflexionen en relación a sus prácticas y su saber, en ese sentido la constitución de los Núcleos Integradores de Problema NIP, proyectos semestrales, la práctica pedagógica y el trabajo de grado constituyen un medio de acercamiento a tal intención, entretanto la investigación se ubica como el ejercicio que da forma y encamina estas apuestas.

Desde esta posición el desarrollo de una monitoria de investigación pone en escena el trasegar al interior de la licenciatura en función de la búsqueda de posibilidades al interior de los distintos ejes problémicos, de modo que tal ejercicio constituye una puerta a la constitución de insumos que posicionan el quehacer del futuro profesional de la enseñanza de la biología generando experiencia académica y pedagógica.

Entre tanto, surge el proyecto Estrategia pedagógica para la enseñanza de la vida y lo vivo a partir de las colecciones biológicas: Hacia la configuración del Museo Pedagógico de Biología. Cuyos propósitos se centran en la sistematización de prácticas pedagógicas, la construcción noción del Museo pedagógico de Biología y el diseño de la estrategia pedagógica.

Estas intencionalidades dialogan en función del reconocimiento del marco histórico y procedimental de las colecciones biológicas existentes al interior del Departamento, en consecuencia, tienen lugar el análisis por los saberes, propuestas y objetos que tienen lugar desde tales escenarios; este talante pone en escena las prácticas que llevan al cabo los maestros en formación y en ejercicio. De tal suerte a esta labor se configura no solo en una posibilidad de enriquecimiento formativo, sino que dialoga al entendimiento de los motivantes, elementos y conceptualizaciones que atañen a los maestros de Biología.

Desde esta comprensión es válido entender la investigación como ejercicio constructor, reflexivo y dialogante a las aristas de la Enseñanza de la Biología, no en su totalidad sino en el acercamiento a sus dinámicas y conceptos, de este modo la comprensión de la 
Bio - grafía. Escritos sobre la Biología y su Enseñanza. ISSN 2027-1034

Edición Extraordinaria. p.p. $36-43$

Memorias del IX Encuentro Nacional de Experiencias en Enseñanza de la Biología y la

Educación Ambiental. IV Congreso Nacional de Investigación en Enseñanza de la

Biología.

investigación no es retratada como la solución a los problemas de quienes tratan con la enseñanza sino como parte misma de la labor de estos últimos, como el medio de la problematización entre las aristas de la escuela, la formación de los maestros, sus posibilidades y las demás situaciones problema no mencionadas que atañen al quehacer del docente.

\section{DISCUSIONES ALREDEDOR DE UNA ESTRATEGIA PEDAGÓGICA Y LAS COLECCIONES BIOLÓGICAS.}

De acuerdo con Staehle (1999) una característica central del concepto estrategia se marca en la consideración de la asignación de recursos y la ventaja competitiva a largo plazo. Esta significación encuentra similitud con lo propuesto con Valverde (2014) quien afirma que una estrategia requiere de la elección, perfeccionamiento y ejecución adecuada de una serie de técnicas en función de un procedimiento organizado y enfocado a la obtención de una meta preestablecida, nótese en ambos casos la movilización en función de un objetivo expreso, movilización que lleva a la puesta en escena de una serie de detonantes pensados, procesados y desarrollados como pasos hacia el buscado propósito.

Es sugerible la acogida de este tipo de conceptualizaciones en propuestas enmarcadas en procesos administrativos, no obstante, el interés desde tiene lugar la presente consideración se ubica en el quehacer pedagógico, por lo que es menester viabilizar una apuesta que dé lugar a la incorporación no solo de los marcos teóricos de la educación y la pedagogía, sino que los contextualice en el ámbito de las colecciones biológicas y la configuración del Museo de Biología.

Desde la propuesta de Tobón (2004) se lleva a acoger una conceptualización de estrategia como el modo operante y las formas de proceder, es decir la definición de un camino que supone una conceptualización relativa al qué, por qué, para qué, cuánto, dónde y cuándo se quiere obtener, siendo así que lo metodológico no puede ser desprovisto de lo conceptual desde donde tiene lugar la intención frente a los elementos y aquello que se pretende conseguir, de allí que el posicionamiento de estrategia se aleja de las incorporación de elementos dirigidos en exclusividad a la obtención de metas, sino que por el contrario sitúa una intención formativa.

Trayendo a colación el objetivo general de la investigación: "Configurar el Museo Pedagógico de Biología como estrategia pedagógica para la enseñanza de la vida y lo vivo a partir de las colecciones biológicas y la sistematización de las prácticas relacionadas con las mismas, con miras a enriquecer y actualizar las prácticas y saberes en torno del Museo de Biología" (UPN ,2016). Se halla sintonía con una propuesta centrada en la agrupación de acciones dirigidas a la reflexión de las prácticas y la reconfiguración de sus prácticas en participación de los sujetos de saber y los marcos teóricos y procedimentales de la pedagogía.

Las consideraciones alrededor del concepto estrategia, si bien merecen ser disertación, en tal medida este texto se ciñe a presentar la consideración desde donde puede tener 


\section{Bio - grafía. Escritos sobre la Biología y su Enseñanza. ISSN 2027-1034}

\section{Edición Extraordinaria. p.p. 36 - 43}

Memorias del IX Encuentro Nacional de Experiencias en Enseñanza de la Biología y la Educación Ambiental. IV Congreso Nacional de Investigación en Enseñanza de la Biología.

sintonía el proyecto investigativo. Dicho esto, en adelante la preocupación de este texto se centra en la exploración de las posibilidades de una estrategia pedagógica en el lente de las colecciones biológicas.

De acuerdo con el marco teórico del proyecto una estrategia pedagógica es visible como experiencia en función del cuestionamiento de la permanencia de sus prácticas, en consecuencia, sus reconfiguraciones se enmarcan en la problematización alrededor de múltiples posibilidades y nuevas relaciones conducentes a nuevas prácticas.

Esta perspectiva dista de intervenciones como las propuestas realizadas por Sánchez y Barragán (2009) quienes afirman:

"Se concluyó que la estrategia es muy efectiva y apropiada porque el estudiante adquiere diversos aprendizajes relacionados con la escritura, la lectura, la interlocución, el conocimiento del entorno de sus compañeros, el desarrollo de operaciones mentales y otros aspectos que van relacionados con su dimensión afectiva y el fomento de valores importantes para su vida" (p. 1).

Se permite contemplar una estrategia pedagógica alejada de la búsqueda por la adquisición de habilidades, reconociendo así la existencia de una comprensión complejizada que da lugar a la construcción de saberes, pero cuyas intenciones se movilizan y se adaptan desde el ejercicio reflexivo.

Es posible que el lector llame la atención a la necesidad de distinción de cara a las estrategias didácticas, motivante que lleva a la presentación de dos de las apuestas que dialogan desde el desarrollo del proyecto de investigación.

Cammaroto (2003) dirige la atención a las estrategias didácticas en la suposición de un proceso de enseñanza- aprendizaje en presencia o ausencia del maestro, en predisposición por parte del proceso de instrucción el logro por parte del estudiante de una serie de competencias producto del accionar de conductas iniciales. Nótese la extirpación de la figura del maestro, en menoscabo de su necesidad y en su paso limitando el ejercicio de enseñanza a la instruccionalidad, esta lógica que sospecha por la ubicación central la incorporación de los contenidos, como parte del proceso educativo y que a su vez lleva a preguntarse la contemplación didáctica y pedagógica de trasfondo.

En cualquier caso, esta clase de consideraciones requieren de un análisis a profundidad y el reconocimiento de su origen y movilización, que puede contribuir al esclarecimiento de los condicionamientos alrededor de las estrategias.

En contraste una contemplación a la configuración misma de Didáctica, desde la perspectiva de Litwin (1997) deslocaliza el discurso de exclusión del maestro en entendimiento de una Didáctica centrada en las prácticas de enseñanza, motivante que lleva a inferir la posibilidad de una estrategia didáctica centrada en el ejercicio de enseñanza

La conceptualización alrededor de estos términos puede ser esclarecida desde la conceptualización misma de Didáctica y Pedagogía que se ponga en circulación, este detonante lleva a cuestionarse alrededor de la multiplicidad de implicaciones históricas, 


\section{Bio - grafía. Escritos sobre la Biología y su Enseñanza. ISSN 2027-1034}

\section{Edición Extraordinaria. p.p. 36 - 43}

Memorias del IX Encuentro Nacional de Experiencias en Enseñanza de la Biología y la Educación Ambiental. IV Congreso Nacional de Investigación en Enseñanza de la Biología.

conceptuales, procedimentales y propositivas que atañen a cada caso. En consecuencia, se concluye con el llamado al cuestionamiento por el ejercicio del maestro.

Vale la pena considerar otras figuras como las Propuestas pedagógicas, educativas y didácticas; las Orientaciones pedagógicas, las Estrategias de Enseñanza- Aprendizaje y las Estrategias educativas. Quienes en ningún caso son tenidas en cuenta en los párrafos anteriores, pero que sin embargo próximas referenciaciones y acercamientos pueden brindar mayores luces para la diferenciación entre las mismas y entre otras.

Las significaciones de colección biológica no distan de la multiplicidad encontrada por parte de las estrategias. Ejemplo de ello es lo enunciado a continuación.

Mora y Fuentes (2006) dimensiona la comprensión de las colecciones biológicas como inventarios o registros de flora y fauna de algún lugar determinado o que alguna vez ocuparon un espacio temporal. Por su parte Suarez \& Tsutsu (2004) aproxima el lugar de las colecciones biológicas en los ámbitos médicos, ambientales, agrícolas, taxonómicos, sistemáticos, evolutivos, bioquímicos, en el ámbito de la biología molecular, la ecología y el modelaje de la biodiversidad evaluando además las ventajas a nivel económico, así como las facilidades en el intercambio investigativo y la expansión de la labor científica.

En conjunto estas afirmaciones pueden concebir la dimensionalidad de las colecciones biológicas en el acervo físico que representa un conjunto de ejemplares biológicas; sin limitarse a ello, es preciso destacar a la investigación biológica como una de las principales apuestas al interior de las colecciones biológicas, no obstante vale la pena reconocer las posibilidades a nivel educativo, pedagógico y didáctico, que si bien, son presentadas en algunos casos como un elemento adicional e incluso prescindible a la colección, contienen un marco de acciones e intencionalidades que difieren de las prácticas tradicionales.

Es posible una significación del ejercicio educativo a partir del uso de colecciones biológicas alegando la reflexión a prácticas futuras, fomento de la actitud investigativa, el conocimiento de los organismos y acciones como la observación y la participación (Díaz. 2011) por ello las colecciones biológicas aparecen como una apuesta factible en el ámbito escolar conllevado un posicionamiento alrededor de la construcción de los contenidos, su funcionalidad, las aristas que le condicionan y las reflexiones que emergen, lo que en síntesis puede considerarse la inclusión de ejercicio pedagógico en las aplicaciones educativas de las colecciones.

De la mano a ello García, F (2013) evalúa el desaprovechamiento de las colecciones biológicas y el desconocimiento de sus posibilidades en la enseñanza de las ciencias en respuesta a la falta de organización, herramientas o desinterés de los sujetos, por lo que vale la pena preguntarse además de los causantes del desuso y desconocimiento de las colecciones los medios en que pueden ser incluidos y los modos en que el ejercicio pedagógico tiene lugar.

En este punto vale la pena en este caso acercarse a una comprensión de colección biológica con una fuerte vinculación en la investigación biológica pero cuya intencionalidad no finaliza en tal situación, sino que sus posibilidades fluctúan en el ámbito 


\section{Bio - grafía. Escritos sobre la Biología y su Enseñanza. ISSN 2027-1034}

\section{Edición Extraordinaria. p.p. 36 - 43}

Memorias del IX Encuentro Nacional de Experiencias en Enseñanza de la Biología y la Educación Ambiental. IV Congreso Nacional de Investigación en Enseñanza de la Biología.

educativo, susceptible en tal caso a la intervención pedagógica y didáctica.

Por otra parte, al cuestionar la existencia de vínculos de las colecciones biológicas y la biodiversidad. Como lo menciona Rivera (2012). Las colecciones se toman un papel en la educación en el reconocimiento y aprendizaje de la biodiversidad. Situación que da pie a pensar las aristas de la biodiversidad en los ejercicios de enseñanza y aprendizaje, sin embargo, esta última es solo una de las interacciones existentes en la relación biodiversidad - colecciones biológicas.

De acuerdo Vallejo y Acosta (2005) Una colección biológica trasciende de la interpretación del depósito de ejemplares biológicos, su configuración pasa por el talante de patrimonio natural y las entidades museísticas, de modo que las colecciones constituyen una fuente para el conocimiento de la biodiversidad, el análisis evolutivo y ecológico. Este direccionamiento pone evidencia situaciones como las características físicas de las colecciones, la vinculación a la investigación científica y el acceso a la biodiversidad como producto directo de las colecciones, situación que entre tanto denota las múltiples posibilidades de las colecciones.

Estos últimos autores, dirigen la atención a una instancia de interés para el desarrollo del proyecto investigativo: la configuración del Museo pedagógico de Biología, dimensionalidad que tiene lugar desde el reconocimiento de un recorrido histórico de las colecciones (especialmente en el DBI) de la mano al desarrollo investigativo, pedagógico y didáctico de las mismas, en contribución al crecimiento de las ciencias biológicas y una exploración en las pautas alrededor de las colecciones.

Estos funcionamientos, no son más que una corta lectura de lo que fluctúa en el ámbito de las colecciones biológicas, no instante ésta puede ser traducible en una multidimensionalidad en las mismas, es decir con la existencia de múltiples prácticas asociadas a cuanto lugar y opción de conocimiento, como acervo de especímenes o ejemplares, como oportunidad de aprendizaje y como patrimonio nacional, en ese sentido el trabajo con las colecciones dista de interpretaciones únicas y compone un ámbito para la intervención de los marcos teóricos y procedimentales de la Enseñanza de la Biología, la pedagogía y la didáctica.

\section{CONSIDERACIONES PARCIALES.}

El diálogo entre las figuras Colecciones biológicas y Estrategia pedagógica componen un marco de análisis novedoso y en cara al Departamento de Biología fungen como la posibilidad de relectura, análisis y dimensionalidad de las prácticas que tienen lugar a partir de los ejercicios de los docentes, motivante que acerca aporta al esclarecimiento de las condiciones que dinamizan las prácticas y los saberes de las colecciones.

en distanciamiento a la existencia de una realidad única y acabada, el sujeto goza con la posibilidad de reconocer, viabilizar y reconfigurar dando lugar a nuevas prácticas, cuya emergencia pasa por la existencia de condiciones para la creación y el cuestionamiento a la subjetividad. 
Bio - grafía. Escritos sobre la Biología y su Enseñanza. ISSN 2027-1034

Edición Extraordinaria. p.p. 36 - 43

Memorias del IX Encuentro Nacional de Experiencias en Enseñanza de la Biología y la

Educación Ambiental. IV Congreso Nacional de Investigación en Enseñanza de la Biología.

La configuración de la figura de Museo pedagógico de biología establece una nueva instancia en las relaciones que existen entre las colecciones biológicas y el museo, motivante que da lugar a un ejercicio en función de las posibilidades del DBI.

\section{BIBLIOGRAFIA}

Cammaroto, A. Martins, F. y Paella, S. (2003). Análisis de las estrategias instruccionales empleadas por los profesores del área de matemática: Caso: Universidad Simón Bolívar. Sede' Litoral [Articulo en línea]. Investigación y Postgrado. abr.2003,"vol.18,"no.1

Diaz, L. Meza, M. \& Montaño, M. (2012). La colección entomológica CEBUC y su potencial como colección de referencia de insectos acuáticos. Recuperado de ttp://ilam.org/LAMDOC/sobi/Protocolo-para- la-conservacion- manejo-coleccionesbiologicas.pdf

García F, (2013) Las colecciones biológicas como estrategia didáctica generadora de aprendizajes significativos en ciencias naturales y educación ambiental.

Litwin, E. (1997). Configuraciones Didacticas, Las. Paidc"s.

Mora, L. y Fuentes, H. (2006) El laboratorio de Colecciones Biológicas de la Unviersidad del Mar: perspectivas de una colección regional. Ciencia y Mar 10, (28), 34-37

Sánchez y Barragán (2009) B. ANÁLISIS DE LA ESTRATEGIA "DIARIOS DE MUÑECOS VIAJEROS" RESPECTO A CRITERIOS DIDÁCTICOS PARA TRANSFORMAR LA PRODUCCIÓN ESCRITA EN BÁSICA PRIMARIA

Staehle (1999) : gestión. 8ª edición. Vahlen, Munich

Suarez, A. V., \& Tsutsui, N. D. (2004). The value of museum collections for research and society. BioScience, 54(1), 66-74

Tobón, R. (2004). Estrategias comunicativas en la educación: hacia un modelo semiótico pedagógico. Universidad de Antioquia.

Universidad Pedagógica Nacional (2016) Estrategia pedagógica para la enseñanza de la vida y lo vivo a partir de las colecciones biológicas: Hacia la configuración del Museo Pedagógico de Biología. [Documento de uso interno]

Vallejo, M. y Acosta, A. (2005) Aplicación de indicadores de conocimiento sobre biodiversidad para el diagnóstico y comparación de colecciones biológicas. Nova, $3,(4), 1-10$.

Valverde, Y. (2014). Lectura y escritura con sentido y significado, como estrategia de pedagógica en la formación de maestros. Revista Fedumar Pedagogía y 
Bio - grafía. Escritos sobre la Biología y su Enseñanza. ISSN 2027-1034

Edición Extraordinaria. p.p. $36-43$

Memorias del IX Encuentro Nacional de Experiencias en Enseñanza de la Biología y la Educación Ambiental. IV Congreso Nacional de Investigación en Enseñanza de la Biología.

Educación, 1(1), 71-104.

Villamizar, J. A (2011). Diseño de una estrategia pedagógica para la enseñanza de la biología de los organismos, a través de las quecas (Scaptocoris sp., Cydnidae) (Master dissertation, Universidad Nacional de Colombia 